\title{
A Novel Technique to Validate Dosimetry for Single-Isocenter Multiple-Target VMAT Stereotactic Radiosurgery
}

\section{Yulin Song1*, Miao Zhang1, Laszlo Voros ${ }^{1}$, Xiaoli Tang1, Ziad Saleh¹, Weixing Cai' ${ }^{1}$, Jeho Jeong1, Boris Mueller², Borys Mychalczak²}

${ }^{1}$ Department of Medical Physics, Memorial Sloan Kettering Cancer Center, New York, USA

${ }^{2}$ Department of Radiation Oncology, Memorial Sloan Kettering Cancer Center, New York, USA

Email: *songy@mskcc.org

How to cite this paper: Song, Y.L., Zhang, M., Voros, L., Tang, X.L., Saleh, Z., Cai, W.X., Jeong, J., Mueller, B. and Mychalczak, B. (2021) A Novel Technique to Validate Dosimetry for Single-Isocenter Multiple-Target VMAT Stereotactic Radiosurgery. International Journal of Medical Physics, Clinical Engineering and Radiation Oncology, 10, 59-68.

https://doi.org/10.4236/ijmpcero.2021.1020 06

Received: March 25, 2021

Accepted: May 21, 2021

Published: May 24, 2021

Copyright (๑) 2021 by author(s) and Scientific Research Publishing Inc. This work is licensed under the Creative Commons Attribution International License (CC BY 4.0).

http://creativecommons.org/licenses/by/4.0/

\begin{abstract}
Each year, 170,000 cancer patients in the United States develop brain metastases. Many of them present with multiple small lesions. Historically, Linac-based stereotactic radiosurgery (SRS) was used to treat single solitary brain metastasis with a diameter of less than $3.0 \mathrm{~cm}$, while whole brain radiation therapy (WBRT) was used to treat multiple brain metastases mainly as palliative therapy. Evidence-based practices reveal that WBRT results in poor treatment outcomes, with high local recurrence rates, decreased cognitive function, and even the onset of dementia. Recently, volumetric modulated arc therapy (VMAT) SRS has been tested as an alternative treatment to WBRT. Owing to its inherent complexity and high risk, it is imperative to perform rigorous testing prior to its clinical implementation. In this paper, we present a novel technique for dosimetry validation of VMAT SRS.
\end{abstract}

\section{Keywords}

Brain Metastasis, VMAT, SRS, WBRT, Film Dosimetry

\section{Introduction}

It is estimated that about $24 \%-45 \%$ of all American cancer patients have brain metastases [1]. As patients survive longer, multiple brain metastases are becoming more prevalent. One theory is that more effective systemic therapies may translate into an increase in brain metastases because chemotherapeutic agents do not penetrate the blood-brain barrier (BBB) [2]. These patients often present with subacute symptoms, such as headaches, nausea, vomiting, seizures, cogni- 
tive changes, motor dysfunction, and photophobia, depending on the location of metastases [3]. Currently, the most effective non-invasive treatment to relieve these symptoms is stereotactic radiosurgery (SRS) [4]. Traditionally, SRS has been used to treat solitary brain metastases. With the advent of volumetric modulated arc therapy (VMAT), there is growing interest in using VMAT SRS to treat multiple brain targets simultaneously with one single-isocenter plan [5]. This technique has several advantages over the conventional one target-one plan paradigm, including shortened treatment time and reduced integral dose to the normal brain tissue. However, the risk associated with VMAT SRS is also elevated if the precision of the delivery system and auxiliary imaging systems is compromised. One contributing factor to this risk is patient rotation-induced geometric misalignment between the actual treatment field aperture and planned field aperture. This arises from the fact that the isocenter of the delivery system can only be placed at one target center. The remaining targets, therefore, become off-isocenter. Dosimetric perturbation by geometric misalignment is negligible for the one target-one plan technique. This is because most brain metastases have a spherical shape, making them relatively immune to patient rotation in terms of delivered dosimetry. However, in the case of VMAT SRS, the magnitude of geometric misalignment is significantly amplified. It is roughly linearly proportional to the distance between the target center and the isocenter, the patient rotation angles, target distribution, and the target size and shape. Therefore, it is crucial to validate the delivered off-isocenter dosimetry for VMAT SRS prior to its clinical implementation. For this purpose, we have developed a novel, but the practical technique that can be easily reproduced in small community radiation oncology centers. In this paper, we present its detailed clinical implementation.

\section{Materials and Methods}

\subsection{Dosimetry Verification Phantom}

We used the commercial STEEV Stereotactic End-to-End Verification Phantom (CIRS, Norfolk, Virginia) for dosimetry validation. The STEEV phantom is made of tissue-equivalent materials. Their linear attenuation coefficients are within 1\% of actual attenuation for soft tissue and bone. The STEEV phantom contains a $64 \times 64 \times 64 \mathrm{~mm}$ film insert in a rectangular brain cavity as shown in Figure 1 . The cubic film insert has a 30 -mm diameter build-in spherical target with $+5 \%$ contrast. Thus, it appears much brighter than the surrounding material in CT scans. This feature greatly facilitates target delineation. A CIRS Precision Cut EBT3 film can be precisely positioned in the center plane of the target and locked by four pins. The pins prevent the film from moving and being misaligned with the insert edges. The film insert is assembled using four pinholes. The STEEV phantom has several external marks, making phantom setup quick, easy, and reproducible. Before inserting the film into the insert, the film should be marked with correct orientation so that the correct film orientation can be restored during film scanning and post-scanning analysis. 


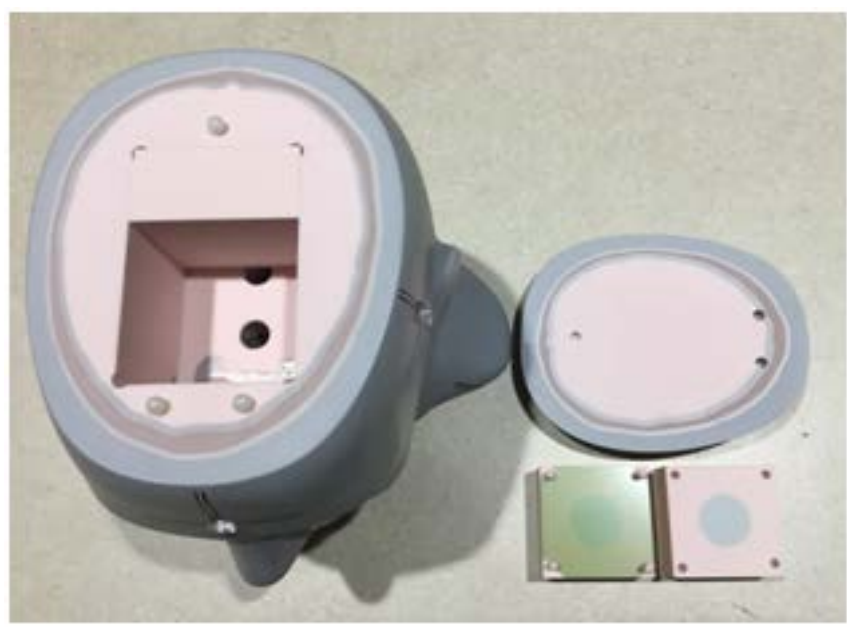

Figure 1. The STEEV stereotactic end-to-end verification phantom. The phantom can be separated into two parts: the inferior part (left) and the superior part (right). The inferior part contains a film insert for dosimetry verification. The two parts can be assembled together by aligning the three pins in the inferior part with the pinholes in the superior part.

\subsection{Treatment Planning with Eclipse}

A VMAT SRS verification plan was computed with Eclipse using our VMAT SRS beam model. The model was specifically developed for small target $(\geq 10$ $\mathrm{mm}$ ) dose calculation for TrueBeam linear accelerators equipped with M120 leaf model (Varian Medical Systems, Palo Alto, California, USA). The verification plan consisted of three spherical planning target volumes (PTV) of $12 \mathrm{~mm}$ in diameter. The central PTV was located at the Linac isocenter. The two peripheral PTVs were located in the coronal and sagittal planes through the isocenter along the film diagonal direction. There are two primary advantages of this particular PTV distribution: 1) increased distances among PTVs and 2) the higher isodose lines being confined within the film. The PTV center-to-center separation was $20 \mathrm{~mm}$ (Figure 2). The verification plan contained two co-planar long arcs and three non-coplanar short arcs (Figure 3). All arcs were equally spaced. The plan prescription dose was $1600 \mathrm{cGy}$, an optimal choice between the response characteristics of EBT3 film and a realistic clinical SRS dose. The volumetric dose calculation was performed with $6 \times$ photons using a special SRS small field beam model developed by our department. The prescription dose was normalized to $100 \%$ isodose line, a paradigm shift from the traditional $80 \%$ normalization methodology.

\subsection{EBT3 Film Calibration}

EBT3 film strips were calibrated with a 7-point geometric scheme. The 7-dose levels were calculated with FilmQA Pro 2017 with the maximum plan dose as input. This method greatly improved the measurement efficiency and minimized the response uncertainty at high dose region. Its basic principle was that each next higher dose level was 1.4 times the previous one. 


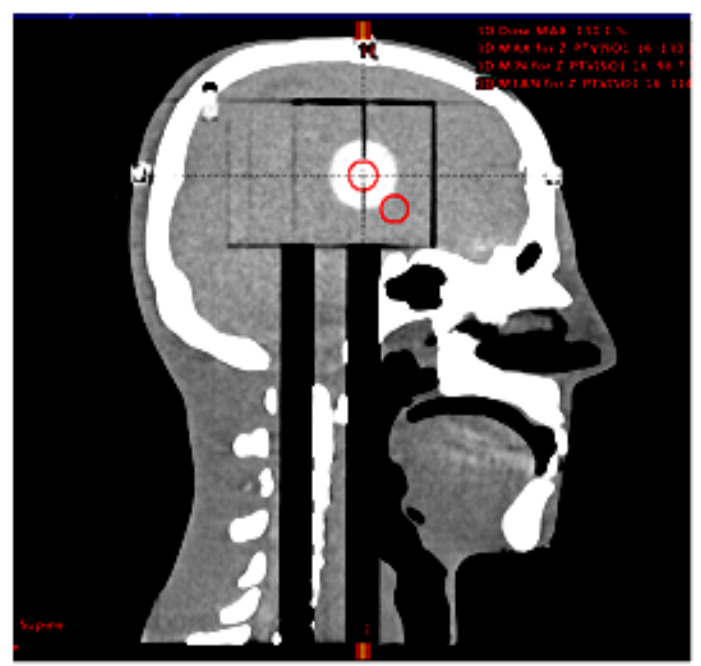

Figure 2. PTV locations in relation to the film. The red solid circles represent the PTVs. The white circular area in the insert center is the built-in target, which was not used by our technique due to its large size.

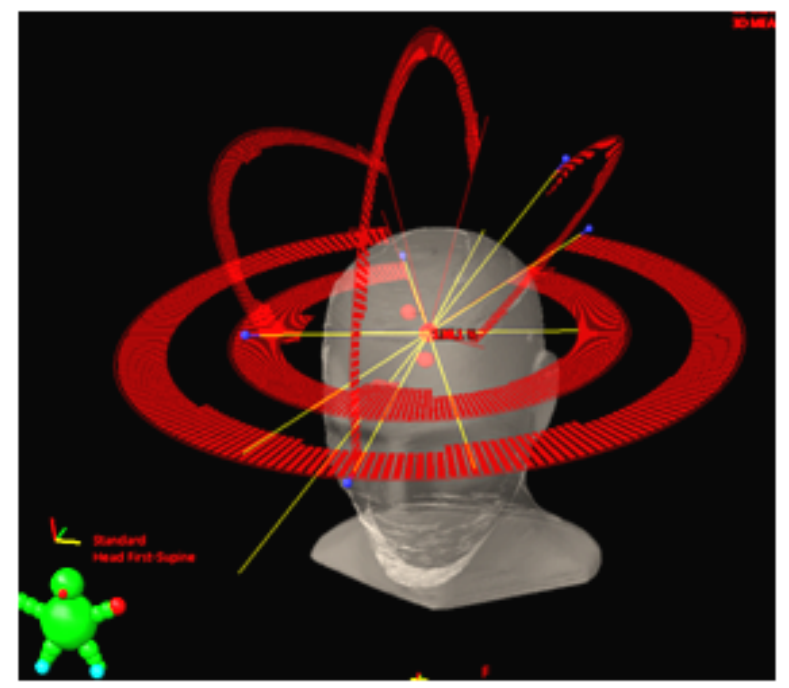

Figure 3. 3D rendition of the PTV distribution and arc arrangement.

Three slabs of $5.0 \mathrm{~cm}$ solid water were used in the film calibration. The calibration film was sandwiched in between two slabs of $5.0 \mathrm{~cm}$ solid water at 100 $\mathrm{cm}$ away from the Linac radiation source. The jaw and MLC were set to $3.0 \times 3.0$ $\mathrm{cm}$. The calibration film strips were exposed according to the pre-determined 7 dose levels. An unexposed film strip was reserved and used as zero dose exposure. In addition, one piece of film was delivered to $1600 \mathrm{cGy}$ with a field size of $3.0 \times 3.0 \mathrm{~cm}$ at the same depth for the purpose of calibration validation.

\subsection{Film Dosimetry Measurement}

The film dosimetry was performed on the latest model TrueBeam Linac equipped with a 6-degrees of freedom (6-DOF) couch (Varian Medical Systems, Palo Alto, CA, USA). The 6-DOF couch is capable of achieving a precision of $0.1 \mathrm{~mm}$ in 
translational shifts and $0.1^{\circ}$ in rotational shifts. The TrueBeam Linac also has the latest version of the Optical Surface Monitoring System (OSMS). It is an ideal choice for performing both conventional radiotherapy and Linac-based SRS. Prior to film dosimetry measurement, the STEEV SRS phantom was initially aligned with the OSMS to within an accuracy of $1.0 \mathrm{~mm}$ in translational shifts and $1.0^{\circ}$ in rotational shifts and then further fine-aligned with the on-board cone beam CT (CBCT). The CBCT scan was acquired with a high-quality head protocol. The slice thickness was $1.0 \mathrm{~mm}$ with a matrix size of $512 \times 512$. A suitable volume of interest (VOI) was selected for the $3 \mathrm{D} / 3 \mathrm{D}$ registration. $\mathrm{Mu}-$ tual information-based auto-registration was then performed for phantom shift calculation. After the shift corrections were applied, a second CBCT scan was acquired to confirm the accuracy of the phantom setup. The plan was then delivered in clinical mode.

\subsection{Film Dosimetry Analysis}

After a 24-hour color stabilization period, the exposed EBT3 film strips were scanned with a resolution of $450 \mathrm{dpi}$ and initially processed with one-scan protocol using FilmQA Pro 2017. The choice of scanning resolution was crucial because 450 dpi provided target localization accuracy up to $0.056 \mathrm{~mm}$. The dose maps were exported in TIFF format and then analyzed with our in-house film dosimetry analysis software.

\section{Results}

Figure 4 shows the planned (right) and measured film (left) dose distributions in the coronal plane, respectively. The red crosshair in the middle of the PTV1 dose distributions was the Linac isocenter. The grid resolution was $2.0 \mathrm{~mm}$. As EBT3 films were very noisy in the high dose region, a $5 \times 5$ smoothing filter was applied to the dose maps in order to enhance the signal-to-noise ratio (SNR) of the data. Figure 5 is the overlay of the two dose distributions. The solid curve

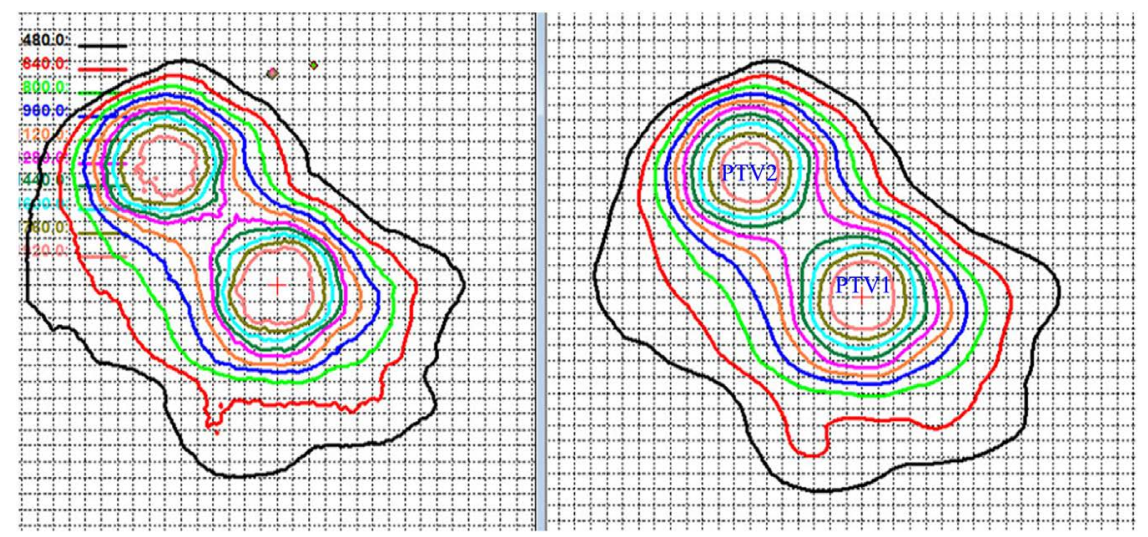

Figure 4. The Planned (right) and measured film (left) dose distributions in the coronal plane with a grid resolution of $2.0 \mathrm{~mm}$. The highest isodose line displayed is $120 \%$ (orange color) and the lowest isodose line is $30 \%$. Each isodose line represents a $10 \%$ dose difference. 


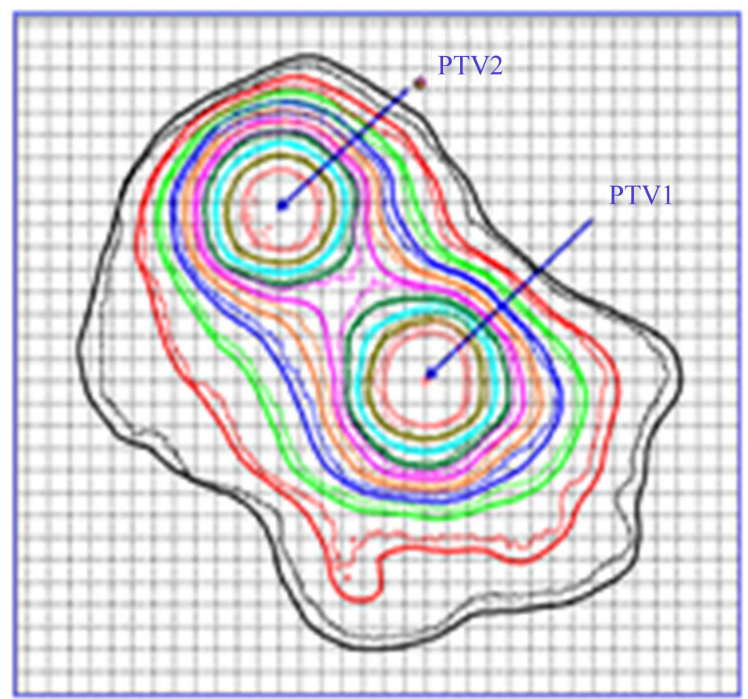

Figure 5. The overlay of planned dose distrition (solid line) and measured film dose distribution (dotted line).

represents the planned dose distribution and the dotted curve represents the measured film dose distribution. The two dose distributions reveal a high degree of dosimetric congruence, in particular, in the high dose region of clinical interest inside the targets. In the area outside the targets, as indicated by the $100 \%$ isodose line (cyan color), the two dose distributions still match quite well. Even in the area distant to the targets (near film edges), the geometric agreement between the two distributions is still within our institutional tolerance. The mean spatial separation between the two distributions is less than $1.0 \mathrm{~mm}$, with the measured film dose distribution being slightly smaller at the lower isodose lines (lower than 40\%). This is consistent with our past observations in film dosimetry measurements for single target SRS plans calculated with iPlan (BrainLab, Germany).

Figure 6 shows representative dose profiles for PTV1 along the $\mathrm{x}$-axis (left) and y-axis (right), respectively. The white line is the film dosimetry. The white vertical lines indicate the film edges, where the measured isodose lines stop abruptly. Even though the target is quite small, an excellent profile agreement is achieved inside the target. Figure 7 shows representative dose profiles for PTV2. In this case, PTV2 is $20 \mathrm{~mm}$ away from the Linac isocenter. Again, we observe an excellent agreement between the two profiles, even in the low dose regions at the film edges.

Figure 8 shows the planned (right) and measured film (left) dose distributions in the sagittal plane, respectively. Figure 9 is the overlay of the two dose distributions. The sagittal plane demonstrates the same characteristics of dosimetric and geometric congruence as the coronal plane. Figure 10 shows representative dose profiles for PTV1 along the $\mathrm{x}$-axis (left) and y-axis (right), respectively. Figure 11 shows representative dose profiles for PTV3 along the $\mathrm{x}$-axis (left) and $y$-axis (right), respectively. 

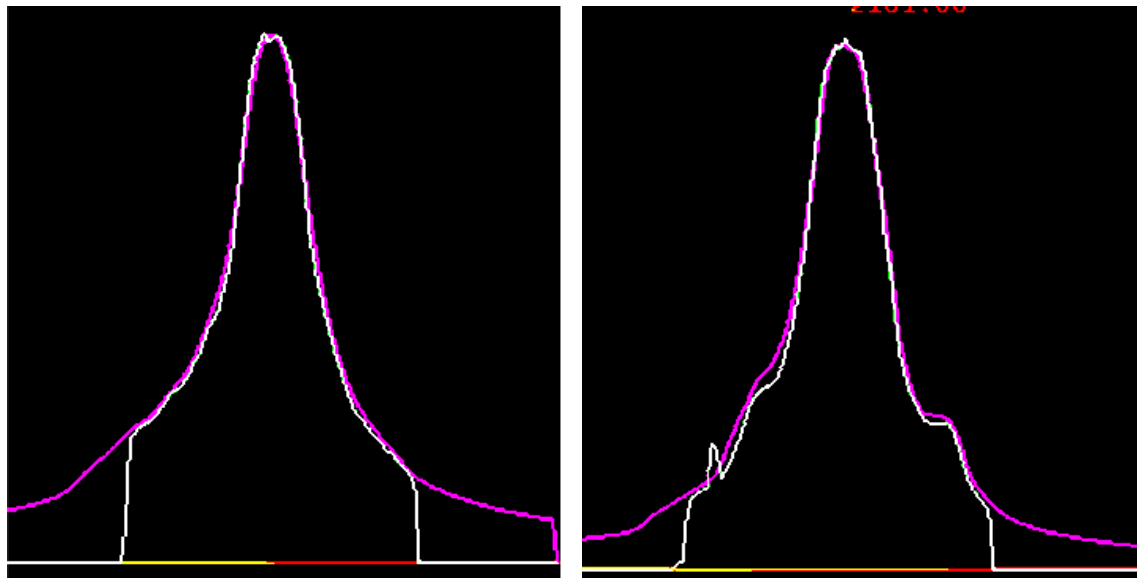

Figure 6. Representative dose profiles for PTV1 along the x-axis (left) and y-axis (right) in the coronal plane. The small spike near the film edge for the $y$-axis profile (right) was caused by the film orientation mark on the film.
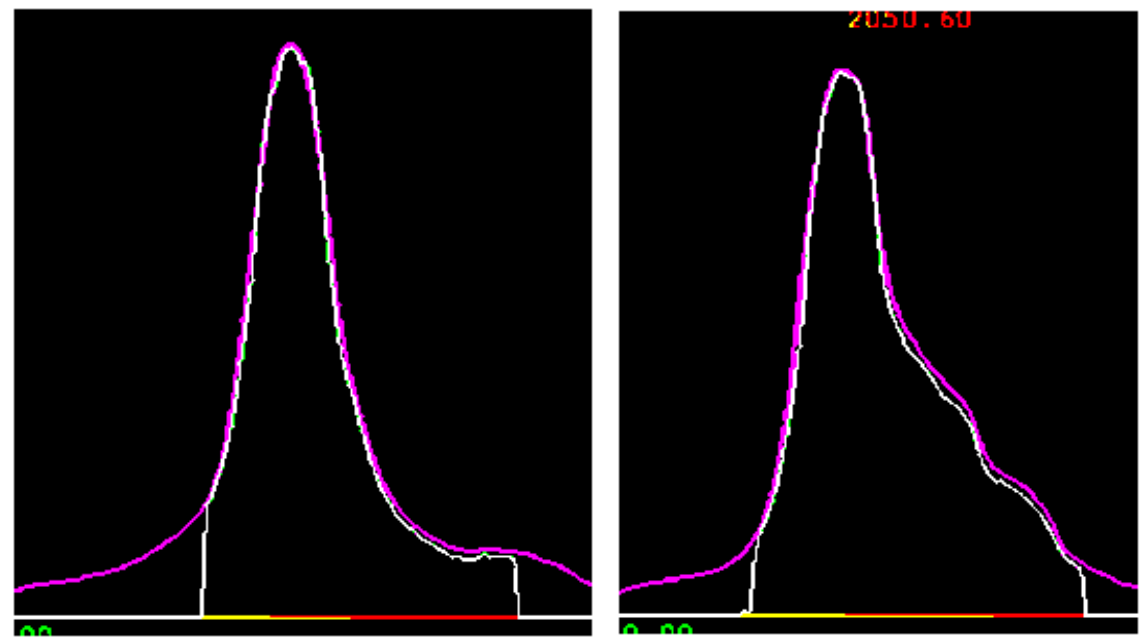

Figure 7. Representative dose profiles for PTV2 along the x-axis (left) and y-axis (right) in the coronal plane. A good agreement between the plan and film dosimetry was also achieved.

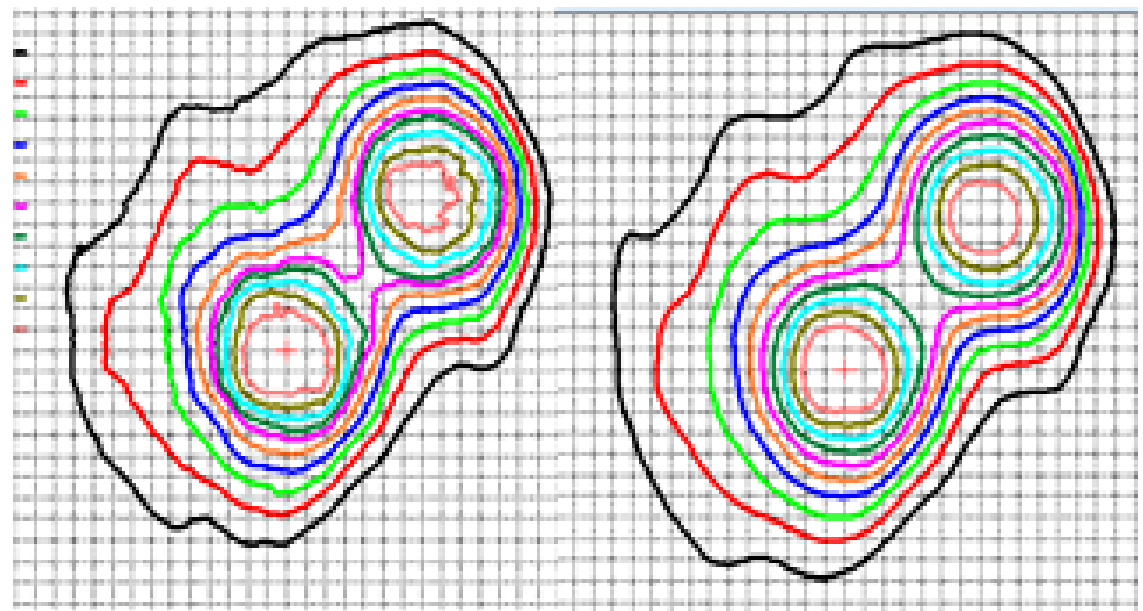

Figure 8. Planned (right) and measured film (left) dose distributions in the sagittal plane. 


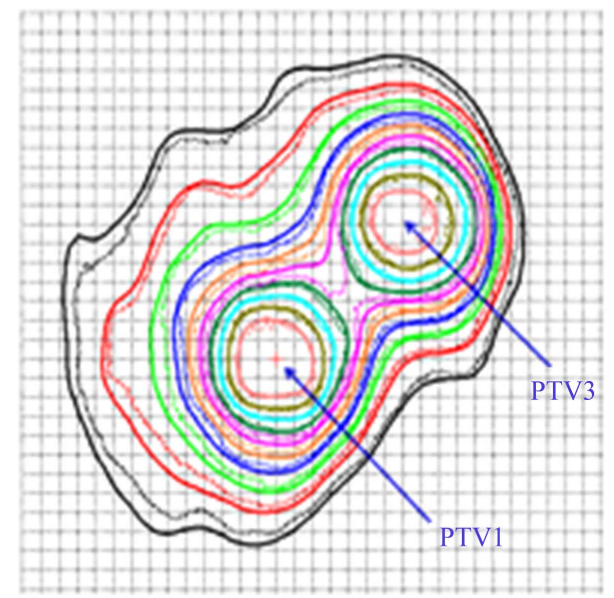

Figure 9. The overlay of planned dose distribution (solid line) and measured film dose distribution (dotted line) in the sagittal plane.
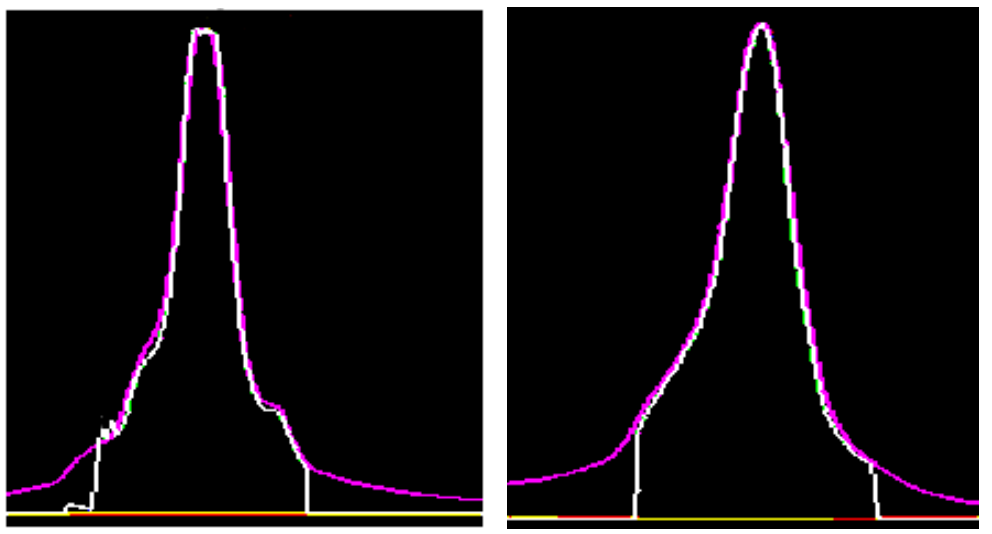

Figure 10. Representative dose profiles for PTV1 along the x-axis (left) and y-axis (right) in the sagittal plane.
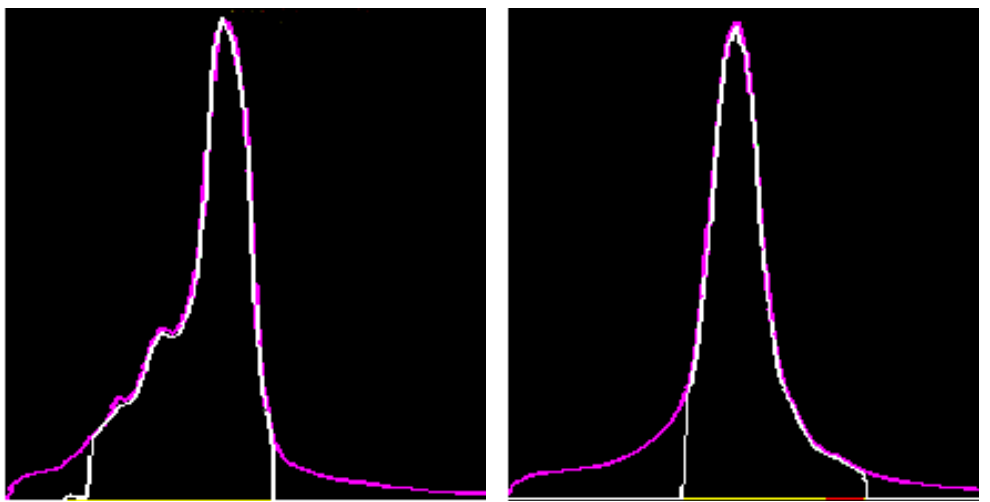

Figure 11. Representative dose profiles for PTV3 along the x-axis (left) and y-axis (right) in the sagittal plane.

Table 1 (upper) shows the mean dose differences between planned and measured film dose distributions in $90 \%$ of the region of the interest (ROI). The dosimetric significance of the $90 \%$ ROI is that it is slightly larger $(\sim 0.75 \mathrm{~mm})$ than the PTV radius and covers the most clinically relevant part of the treatment 
Table 1. Mean dose differences (upper), $\gamma$ pass rates (middle), and target localization uncertainties (lower).

\begin{tabular}{|c|c|c|c|}
\hline ROI & Mean Film Dose (cGy) & Mean Eclipse Dose (cGy) & Dose Diff (\%) \\
\hline $90 \%$ (Coronal) & 1788.34 & 1757.58 & $1.750 \%$ \\
\hline $90 \%$ (Sagittal) & 1753.08 & 1748.85 & $0.242 \%$ \\
\hline ROI & Plan & \multicolumn{2}{|c|}{$\gamma$-Pass Rate $2 \%(2 \mathrm{~mm})$} \\
\hline $90 \%$ & Coronal & \multicolumn{2}{|l|}{$99.86 \%$} \\
\hline $90 \%$ & Sagittal & \multicolumn{2}{|l|}{$99.48 \%$} \\
\hline \multicolumn{2}{|r|}{ Plane } & \multicolumn{2}{|c|}{ Uncertainty (mm) } \\
\hline \multicolumn{2}{|c|}{ Anterior-Posterior } & \multicolumn{2}{|l|}{0.1236} \\
\hline \multicolumn{2}{|c|}{ Right-Left } & \multicolumn{2}{|l|}{-0.3133} \\
\hline \multicolumn{2}{|c|}{ Superior-Inferior } & \multicolumn{2}{|l|}{-0.5511} \\
\hline
\end{tabular}

volume. In this dosimetry verification test, the mean dose difference for the two planes is $0.996 \%$. Table 1 (middle) lists the $\gamma$ pass rates calculated in the $90 \%$ ROI with criteria of $2 \%$ and $2.0 \mathrm{~mm}$. Table 1 (lower) lists the target localization uncertainties in the three anatomical directions, determined from the film dosimetry analysis. Our institutional tolerance is less than $1.0 \mathrm{~mm}$.

\section{Discussion and Conclusions}

Currently, the primary treatment options for patients with brain metastases include systemic therapy, surgery, stereotactic radiosurgery, WBRT, or some combination of these. For patients with multiple small lesions $(\leq 20,<2.0 \mathrm{~cm})$ or deeply seated lesions and poor performance status, the single-isocenter multiple-target VMAT SRS is an excellent treatment option. An increasing volume of clinical evidence suggests that multiple-target VMAT SRS offers significant benefits in both overall survival (OS) and quality of life (QOL) over conventional WBRT. However, the single-isocenter multiple-target VMAT SRS is a complex treatment modality. It requires that every part of the treatment hardware is tested and verified, including the treatment delivery system, auxiliary imaging systems, patient positioning system, and real-time patient tracking system. A carefully designed off-isocenter multiple target film dosimetry is the most critical component of this process.

\section{Acknowledgements}

This research was funded in part by the NIH/NCI Cancer Center Support Grant P30 CA008748.

\section{Conflicts of Interest}

The authors declare no conflicts of interest regarding the publication of this paper. 


\section{References}

[1] Nussbaum, E.S., Djalilian, H.R., Cho, K.H. and Hall, W.A. (1996) Brain Metastases: Histology, Multiplicity, Surgery, and Survival. Cancer, 78, 1791-1788.

https://doi.org/10.1002/(SICI)1097-0142(19961015)78:8<1781::AID-CNCR19>3.0.C $\mathrm{O} ; 2-\mathrm{U}$

https://onlinelibrary.wiley.com/doi/abs/10.1002/\%28SICI\%291097-0142\%28199610 15\%2978\%3A8\%3C1781\%3A\%3AAID-CNCR19\%3E3.0.CO\%3B2-U

[2] Lin, N.U. and Winer, E.P. (2007) The HER2 Paradigm. Clinical Cancer Research, 13, 1648-1655. https://doi.org/10.1158/1078-0432.CCR-06-2478 http://clincancerres.aacrjournals.org/content/13/6/1648

[3] NCCN Clinical Practice Guidelines in Oncology (NCCN) (2018). National Comprehensive Cancer Network. Version 3, February 21, 2018. https://www.nccn.org

[4] Nieder, C, Grosu, A.L. and Gaspar, L.E. (2014) Stereotactic Radiosurgery (SRS) for Brain Metastases: A Systematic Review. Radiation Oncology, 9, Article No. 155. https://doi.org/10.1186/1748-717X-9-155 https://ro-journal.biomedcentral.com/articles/10.1186/1748-717X-9-155

[5] Clark, G.M., Popple, R.A., Young, P.E. and Fiveash, J.B. (2010) Feasibility of Single-Isocenter Volumetric Modulated Arc Radiosurgery for Treatment of Multiple Brain Metastases. International Journal of Radiation Oncology, Biology, Physics, 76, 296-302. https://doi.org/10.1016/j.ijrobp.2009.05.029 https://www.sciencedirect.com/science/article/pii/S0360301609007925 